\title{
Potent NETosis inducers do not show synergistic effects in vitro
}

\author{
MICHAE P. PRUCHNIAK ${ }^{1,2}$, URSZULA DEMKOW ${ }^{1}$
}

${ }^{1}$ Department of Laboratory Diagnostic and Clinical Immunology of Developmental Age, Medical University of Warsaw, Warsaw, Poland ${ }^{2}$ Postgraduate School of Molecular Medicine, Warsaw, Poland

\begin{abstract}
Introduction: NETosis is a process whereby neutrophils release chromatin into the surrounding extracellular matrix to form neutrophil extracellular traps (NETs). Under physiological conditions NETosis can be initiated by a variety of stimuli, including immune complexes, complement activation products, and a milieu of proinflammatory cytokines. Because overproduction of NETs is often related to the promotion or aggravation of autoimmune responses, we decided to assess how simultaneous activation of NETosis by different stimuli affects NET production.

Material and methods: NET formation was initiated by using combinations of three different NETosis inducers: phorbol myristate acetate (PMA), N-formylmethionyl-leucyl-phenylalanine ( $\mathrm{FLLP}$ ) and calcium ionophore (CaI). We measured fluorometry in real time, while microscopic visualisation served as an additional control for NET release. In total, 30 subjects free from infections or chronic diseases were enrolled in this study.

Results: We were able to demonstrate that in all cases NETosis induced by a combination of two stimuli resulted in diminished NETs production when compared to PMA and CaI single stimulations $(p \leq 0.001)$. The only cases in which double stimulation showed similar results to single stimulation were when we compared $f M L P+C a I$ stimulation with $f M L P$ single stimulation. Furthermore, when neutrophils were exposed to all three stimuli NETosis was almost entirely inhibited, compared to any single stimulation $(p \leq 0.001)$.

Conclusions: Our results show that simultaneous stimulation of neutrophils by different NETosis-inducing agents results in diminished formation of NETs compared to a single stimulation. This indicates that cells may possess an internal regulatory mechanism that prevents overgeneration of NETs among healthy people.
\end{abstract}

Key words: autoimmunity, neutrophil extracellular traps, NETosis, neutrophil cross-stimulation.

(Centr Eur J Immunol 2019; 44 (1): 51-58)

\section{Introduction}

Neutrophils are the most abundant type of leukocytes in most mammals. They play an essential role in the innate immune response. In recent years, an understanding of their classic functionality, phagocytosis, and degranulation has been extended to include a unique strategy to fight against bacterial infections - the formation of neutrophil extracellular traps (NETs). The process of NET release is known as NETosis and results in a specific form of cell death, which is different from apoptosis or necrosis, and which leads to the suicidal death of the cell. NETs, at their core, are biological structures containing negatively charged chromatin as their backbone. These structures are ornamented with granule-derived antimicrobial proteins such as myeloperoxidase (MPO), neutrophil elastase (NE), and histones, allowing them to entrap and kill microbes, and thus protecting the host from infections. Conversely, overproduction of NETs can trigger autoimmune reactions, leading to the development of various diseases [1-3]. Therefore, it is important to fully understand the neutrophil activation mechanisms and signalling cascades that cells utilise to create and release NETs.

The over- or under-generation of NETs can have a critical impact on human health. In vivo, neutrophils release NETs upon exposure to a wide range of stimuli, including bacterial fragments, immunological complexes, platelets, and endothelial or even cancer cells [1, 4]. Each of these factors can interact with neutrophils in a unique way. In in vitro studies, NETosis is usually induced by one agent reacting with a specific receptor localised on the cell surface. Little is known about neutrophils and cross-stimulation in the context of NET release. It is known that leukocyte recruitment from circulation to the site of inflammation is a core process in the inflammatory response.

Correspondence: Michał P. Pruchniak, Department of Laboratory Diagnostic and Clinical Immunology of Developmental Age, Medical University of Warsaw, 24 Marszalkowska St., 00-576 Warszawa, Poland, e-mail: michal_pruchniak@yahoo.com Submitted: 05.01.2019; Accepted: 20.02.2019 
This is followed by a second wave of cell recruitment, whereby inflammatory monocytes start to enforce the inflammatory reaction $[5,6]$. Because many of the receptors expressed on the neutrophil surface can be cross-activated in NET triggering processes, the complete pathway of NETosis is still unclear [4, 7]. Serum from patients with autoimmune diseases, rich in proinflammatory cytokines and immunocomplexes, is considered to be a potent NETosis inducer [8]. The amount of NETs generated after incubation with this type of sera is greater than that produced by sera from healthy individuals [9]. In vivo initial overstimulation and subsequent overproduction of NETs potentially leads to the development or aggravation of pre-existing autoimmune disorders [10]. Moreover, excessive formation of NETs has been discovered during sepsis episodes, which is correlated with the development of organ damage. The initial activation of neutrophils by bacterial load followed by stimulation with activated platelets, as well as activated endothelial cells in sepsis, was shown to significantly promote the formation of NETs. In this condition NETs adhere to and activate the vascular endothelium, which ultimately leads to endothelial cell damage $[2,11]$. Therefore, it is crucial to investigate the impact of cross-activation of neutrophils in the context of NET generation, both in health and disease.

In physiological conditions NETosis can be initiated by both natural and synthetic agents. The most potent naturally occurring NETosis inducers are bacterial components, mainly lipopolysaccharide (LPS), lipophosphoglycan (LPG), and M1 protein. Other non-bacterial factors including interleukin 8 (IL-8), tumour necrosis factor alpha (TNF- $\alpha$ ), and a variety of immunological complexes can promote NET formation both in vivo and in vitro. In our experiments, we used three NETosis inducers: phorbol myristate acetate (PMA), $N$-formylmethionyl-leucyl-phenylalanine (fMLP), and calcium ionophores (CaI) $[2,5]$. Each of the used stimuli can activate a distinctive intracellular signalling pathway that leads to NET production (Fig. 1).
The objective of the present study was to explore NET formation through activation of distinctive ROS-inducing intracellular cascades leading to NETosis. Inside the body cells are often surrounded by a milieu of various biological agents that can activate them simultaneously. In this instance we are particularly curious about potential synergistic effects that distinctive NETosis-inducing agents can have upon neutrophils in vitro. This study may help us obtain insights into the NET biology and lay the foundation for future research on NET formation and inhibition.

\section{Material and methods}

\section{Study group}

Thirty volunteers, including males and females, were enrolled for this study. Their ages ranged between 17 and 46 years (median with SD: $36.5 \pm 8.56$ ). All subjects underwent a self-assessment test. Volunteers who passed the acceptance criteria were free from chronic conditions and infectious diseases. Informed, written consent was obtained from all participants. All blood samples were processed up to 90 minutes post blood collection. The study protocol was approved by the Bioethics Committee of the Medical University of Warsaw.

\section{Blood collection and neutrophil isolation}

Blood from healthy volunteers was drawn from the cephalic vein into collection tubes containing 3.2\% (0.109 M) buffered sodium citrate. Whole blood was centrifuged at $160 \mathrm{~g}$ for $10 \mathrm{~min}$ at room temperature (RT), and the plateletrich plasma was discarded. The remaining blood fraction was diluted with phosphate buffered saline PBS (P4417, Sigma-Aldrich), layered on Histopaque 1077 (10771, Sigma-Aldrich), and centrifuged for $30 \mathrm{~min}$ at $420 \mathrm{~g}$. The interphase containing lymphocytes was discarded and the granulocyte rich fraction was washed with RPMI 1640 medium (R8758, Sigma-Aldrich) prior to being loaded on a poly-

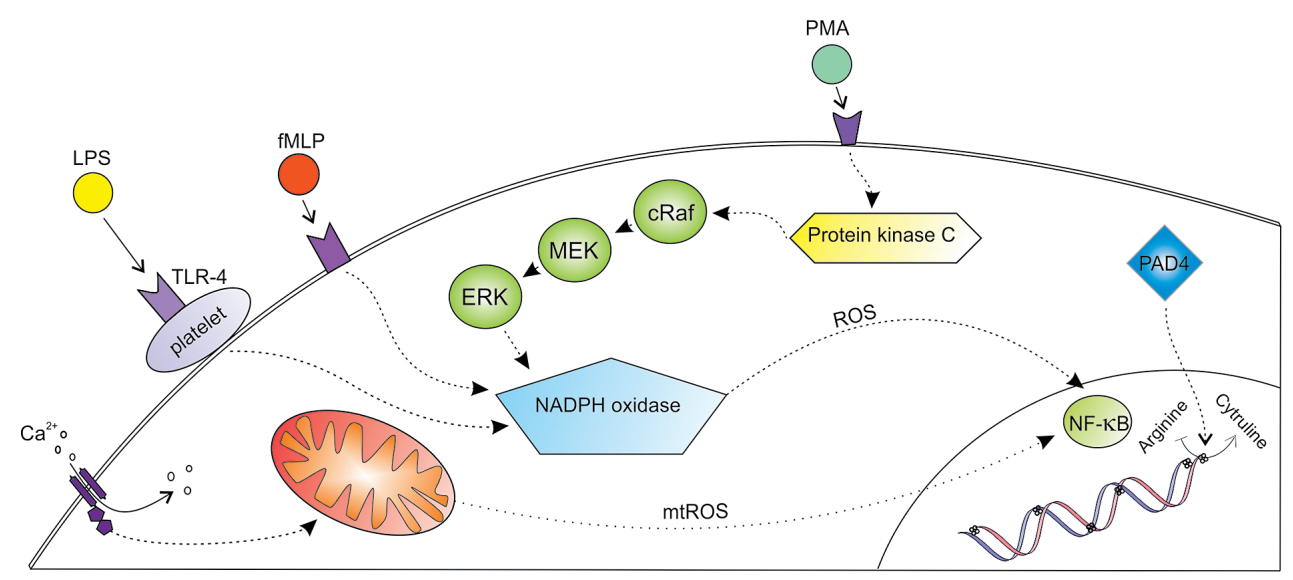

Fig. 1. Selected NETosis inducers and their intracellular activation pathways 
vinyl alcohol gradient (734892424, POCH). After 20 minutes of sedimentation the upper phase containing granulocytes was collected, washed three times by adding PBS (by centrifugation: $300 \mathrm{~g}, 10 \mathrm{~min}, \mathrm{RT}$ ), and suspended in RPMI 1640 medium after the last wash. The isolation procedure was completed within three hours of blood collection.

\section{Samples quality}

The purity of the isolated PMNs was ascertained by immunophenotyping with CD15-FITC (fluorescein isothiocyanate), CD33-PE (phycoerythrin), and CD45-PECy5.5 (phycoerythrin-cyanine 5.5). Viability tests were based on Trypan Blue (93595, Sigma-Aldrich) exclusion and Anexin V/Propidium Iodide (PI) staining (556547, BD Biosciences). Flow cytometry analyses were performed using a Cytomics FC 500 cytometer (Beckman Coulter).

\section{Fluorometric quantification of NET-DNA release}

$1 \times 10^{5}$ neutrophils were seeded into each well of a 96well black plate (FIA - plate, Greiner). Cells were stained with $1 \mu \mathrm{M}$ SYTOX green (S7020, Thermo Fisher Scientific), a fluorescent dye that is impermeant to live cells and has a high-affinity to nucleic acid. Subsequently, cells were allowed to settle for 20 minutes and then stimulated with either 100 nM PMA (79346, Sigma-Aldrich), $1 \mu \mathrm{M}$ fMLP (F3506, Sigma-Aldrich), or $4 \mu \mathrm{M}$ CaI (C7522, SigmaAldrich) or respective mix of agents (PMA and fMLP; PMA and CaI; fMLP and CaI; fMLP, CaI, and PMA). The fluorescence of NET-bound SYTOX Green (excitation $\lambda$ : $488 \mathrm{~nm}$, emission $\lambda$ : $510 \mathrm{~nm}$ ) was measured every $30 \mathrm{~min}$ utes for a period of three hours at $37^{\circ} \mathrm{C}$ using a FLUOstar ${ }^{\circledR}$ Omega multi-mode microplate reader (BMG Labtech, Ortenberg, Germany) and Omega Data Analysis software. DNA release values are presented as the relative fluorescence units (RFU), which reflect the free DNA concentration.

\section{Visualisation of NET-DNA release}

$1 \times 10^{5}$ neutrophils suspended in $400 \mu$ of RPMI1640 were seeded on culture slides (Nunc ${ }^{\mathrm{TM}}$ Lab-Tek $^{\mathrm{TM}}$ II Chamber Slide ${ }^{\mathrm{TM}}$, Thermo Fisher Scientific) and allowed to settle for $30 \mathrm{~min}$ at $37^{\circ} \mathrm{C}$ and then stimulated with either $100 \mathrm{nM}$ PMA, $1 \mu \mathrm{M}$ fMLP, $4 \mu \mathrm{M} \mathrm{CaI}$, or a respective mix of agents. Samples were stimulated for three hours at $37^{\circ} \mathrm{C}$. After incubation samples were fixed with $4 \%$ paraformaldehyde (715400427, POCH) and then stained with $1 \mu \mathrm{M}$ SYTOX Green to visualise NET structures. Samples were analysed using a Nikon Eclipse E200 fluorescence microscope. NETs and remaining cells were observed using a $40 \times$ magnification objective, and images were captured on a Nikon DS-Filc digital camera.

\section{Statistical analysis}

Excel software (Microsoft Office) or GraphPad InStat (Graphpad Inc.) were used for all statistical analyses.
The D'Agostino \& Pearson normality test was used to determine the data distribution. For parametric and normally distributed data, Student's $t$-test was used. For non-parametrically distributed data, the Mann-Whitney test was applied. The statistical significance was calculated as followed: ns $p>0.05, * p \leq 0.05, * * p \leq 0.01$, ***p $\leq 0.001$.

\section{Results}

\section{Sample characteristics}

For our experiment we used neutrophils isolated from peripheral blood of healthy individuals. The mean neutrophil count per sample was $88.81 \%$ with SD of $5.48 \%$, while viability was $94.82 \% \pm 2.59 \%$ based on Anexin V/PI staining (data not shown).

\section{Combinations of PMA, fMLP, and CaI decrease NET release}

In order to investigate whether classical NETosis inducers can act synergistically and increase the NET formation in vitro, we stimulated neutrophils with various combinations of stimuli. Quantitative evaluations of NET formation by real-time fluorometric analysis are shown in Figure 2. A fluorescent microscopy visualisation was performed in parallel with the kinetic readings (Fig. 3).

As previously noted, NET formation depends on the stimulus used to trigger the process. To further elucidate the differences in NET release, we compared the kinetics of induced NET extrusion where more than one stimulus was used. When neutrophils were stimulated with a mixture of PMA and fMLP, the amount of released DNA was significantly lower in comparison with single PMA stimulation $\left.{ }^{*}\right)$. However, no significant difference was observed when the data were compared to stimulation with fMLP only. Activation of cells with the second combination of NETosis inducers, PMA and CaI, resulted in significantly lower DNA release compared to single stimulation with either PMA $\left(^{*}\right)$ or $\mathrm{CaI}\left({ }^{\dagger}\right)$. Furthermore, a combination of fMLP and $\mathrm{CaI}$ resulted in NET release comparable to samples stimulated with fMLP only, which is significantly lower when compared to samples stimulated with CaIonly $\left({ }^{\ddagger}\right)$. However, no significant difference was observed when the data were compared to stimulation with fMLP only. Activation of cells with the second combination of NETosis inducers, PMA and CaI, resulted in significantly lower NET release compared to single stimulation with either PMA $\left(^{*}\right)$ or $\mathrm{CaI}\left({ }^{\dagger}\right)$. Moreover, a combination of fMLP and CaI resulted in NET release comparable to samples stimulated only with fMLP, which was significantly lower when compared to samples stimulated only with $\mathrm{CaI}\left({ }^{\ddagger}\right)$. Furthermore, the microscopic visualisation (Fig. 3) of single-stimulated and co-stimulated neutrophils was performed to confirm the real-time quantification findings. In all cases, the incubation of neutrophils with PMA, 

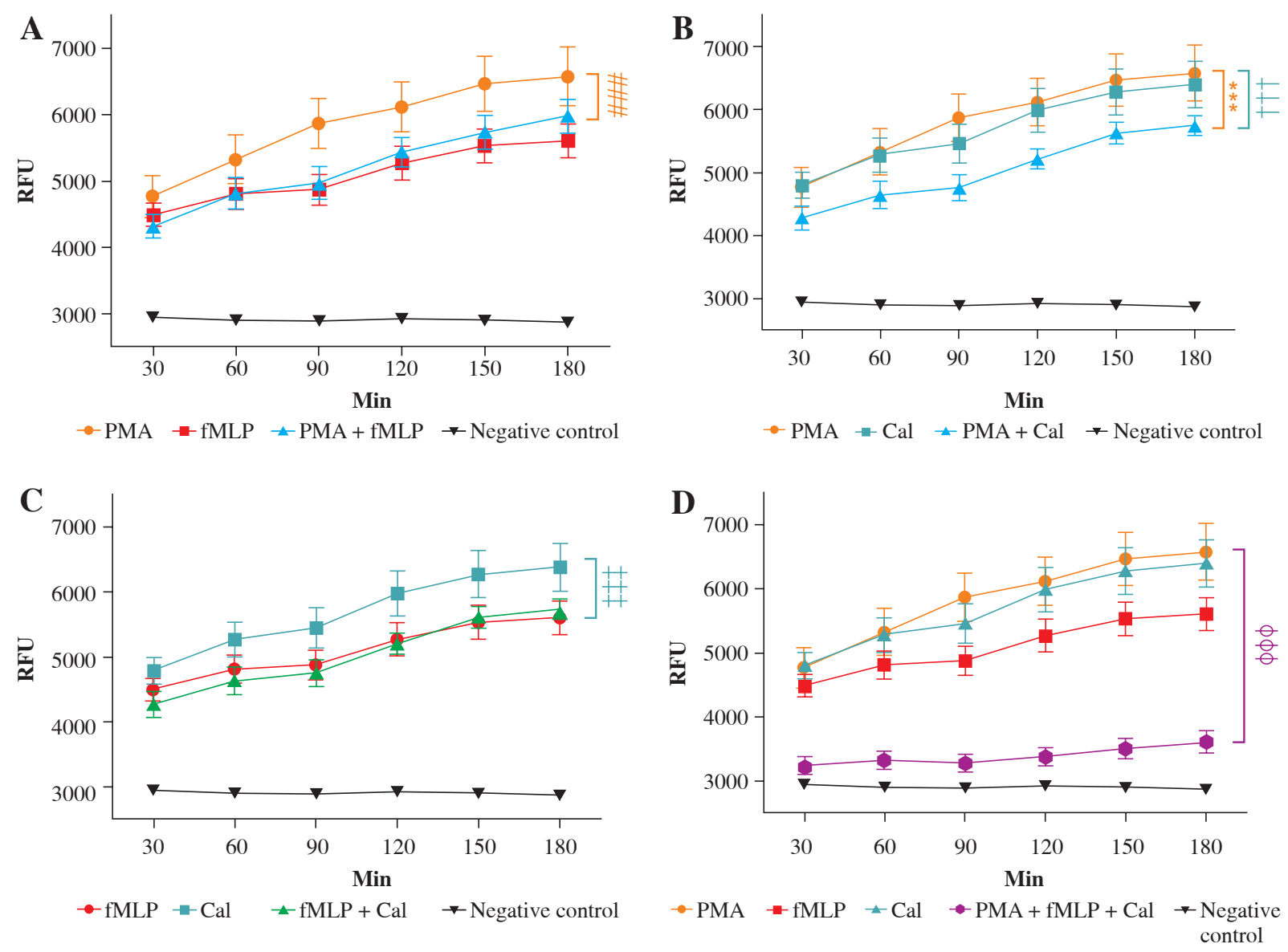

Fig. 2. Real-time quantification of neutrophil extracellular trap (NET) release. Graphs show the real-time kinetics of NETdependent relative fluorescence intensities (RFU) as measured by Sytox Green $(1 \mu \mathrm{M})$ assay. NET generation from human neutrophils was induced by different combinations of phorbol myristate acetate (PMA) (100 nM), $N$-formylmethionyl-leucyl-phenylalanine (fMLP) $(1 \mu \mathrm{M})$, and calcium ionophores (CaI) $(4 \mu \mathrm{M})$, while single-stimulations served as a respective control. Real-time generation of NETs was monitored for 3 hours at $37^{\circ} \mathrm{C}$ with 30 -minute intervals. A) NET release after incubation with a combination of PMA and FMLP; B) NET release after incubation with a combination of PMA and CaI; C) NET release after incubation with a combination of fMLP and CaI; D) NET release after incubation with a combination of PMA, fMLP and CaI. * - significant difference between PMA/fMLP and PMA; * - significant difference between PMA/CaI and PMA $;{ }^{\dagger}-$ significant difference between PMA/CaI and CaI; ${ }^{\ddagger}-$ significant difference between $\mathrm{fMLP} / \mathrm{CaI}$ and $\mathrm{CaI} ;{ }^{\circ}$ - significant difference between PMA/fMLP/CaI and each single stimulus. NET formation was quantified as mean $\pm \mathrm{SD}$, and the statistical significances were calculated as followed: $\mathrm{ns} p>0.05$, $* p \leq 0.05, * * p \leq 0.01, * * * p \leq 0.001$. RFU - relative fluorescence units

fMLP, or CaI resulted in a robust NET release. Double stimulation resulted in less effective NET generation, especially when compared to single-PMA and -CaI samples, reflecting the kinetic data. In all cases activated neutrophils presented classical morphology of a NETotic cells, NETs appeared as irregular cloudy structures with a bright meshwork of threads formed from extracellular DNA. Lobulated nuclei of non-NET-forming cells were used to assess the differences in NET generation. Lastly, when all three stimuli were applied together, the amount of generated NETs was not only significantly lower than each individ- ual stimulus $\left({ }^{\circ}\right)$ but was also significantly lower than each double combination. A microscopic visualisation of stimulated neutrophils revealed that cells exposed to a mixture of PMA, fMLP, and CaI were producing NETs at very low levels throughout the course of incubation (Fig. $3 \mathrm{H}$ ). Moreover, it was observed that these non-NET-forming cells had a different morphology compared to unstimulated controls. The nuclei of triple-stimulated cells appeared swollen with short chromatin threads, while cells from the negative control demonstrated a classical lobular nuclei shape. 


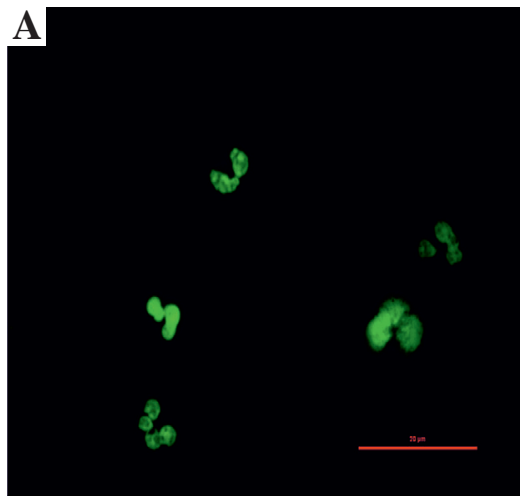

Negative control

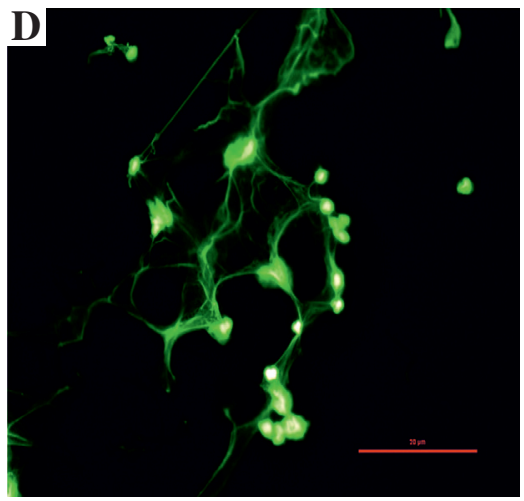

$4 \mu \mathrm{M}$ Cal

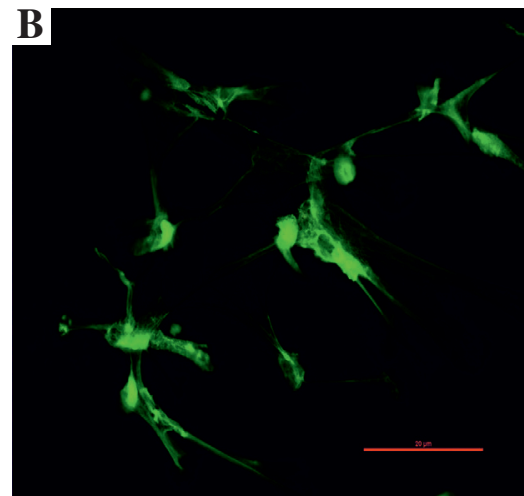

100 nM PMA

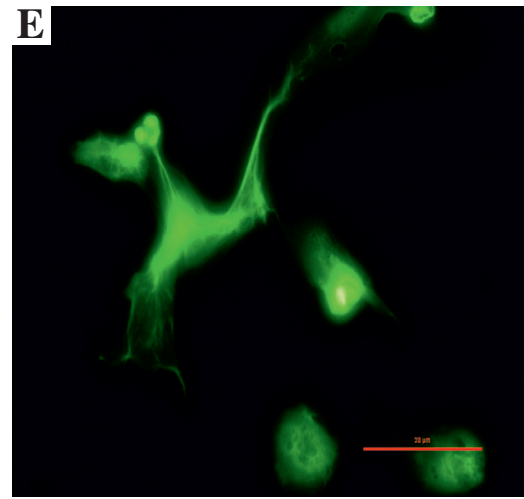

PMA + fMLP

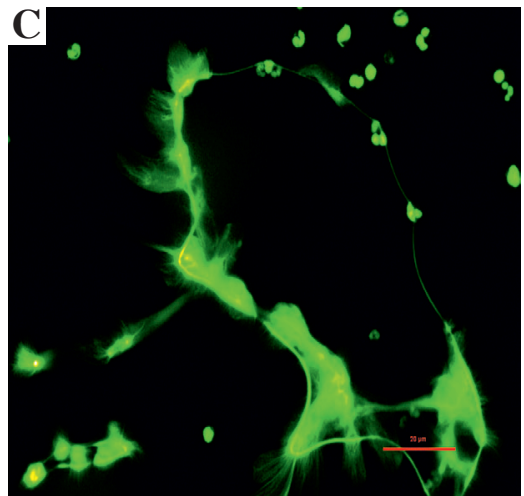

$1 \mu \mathrm{M}$ fMLP

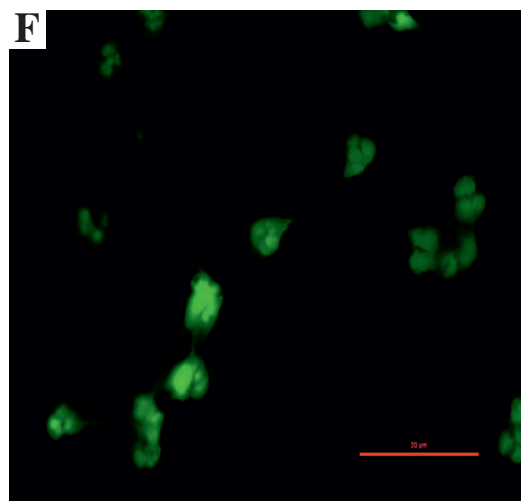

fMLP + Cal

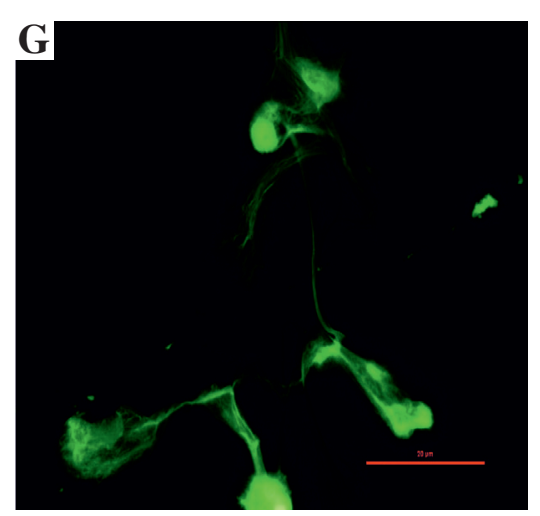

PMA + Cal

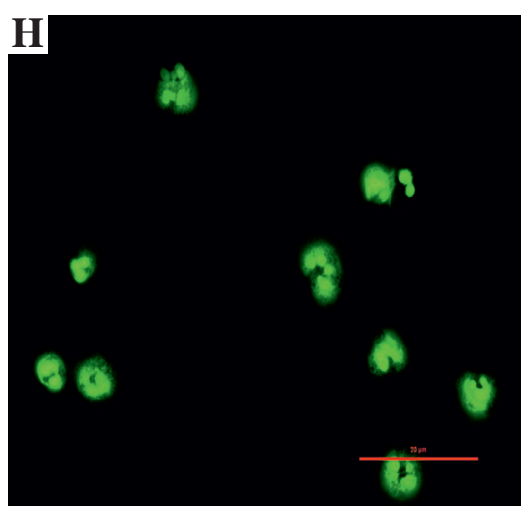

PMA + fMLP + Cal

Fig. 3. Microscopic visualisation of neutrophil extracellular traps. Visualisation of NET release by neutrophils obtained from healthy individuals after 180-minute incubation with a respective stimulating agent or a combination of agents (100 nM phorbol myristate acetate [PMA], $1 \mu \mathrm{M} N$-formylmethionyl-leucyl-phenylalanine [fMLP], or $4 \mu \mathrm{M}$ calcium ionophores [CaI]). After incubation, samples were fixed with paraformaldehyde and a $1 \mu \mathrm{M}$ SYTOX green solution was used to visualise NET structures. Samples were observed at $40 \times$ magnification

\section{Discussion}

Neutrophils and NETosis play a significant role in the innate immune response and act as a first line of defence against invading microorganisms [12]. Although typically beneficial, NETosis can be harmful in certain situations.
In this work we tried to better understand how NET generation is initiated, and whether NETosis inducers can act in synergy to promote more robust NET formation. It was hypothesised that simultaneous activation of different intracellular pathways, which lead to NET generation, would 
result in more robust NETosis. In the current scientific literature there are few studies investigating the synergistic effects of different stimuli on NET production. These studies (details in the discussion) mostly show differences in the NET pre-release events, i.e. ROS generation or histone citrullination after stimulation or pre-incubation with different stimuli $[13,14]$.

Initially, we stimulated neutrophils with a combination of PMA and fMLP. PMA is an activator of protein kinase $\mathrm{C}$ (PKC), which strongly promotes NET generation by increasing intracellular ROS levels through activation of nicotinamide adenine dinucleotide phosphate (NADPH) oxidase via the Raf-MEK-ERK pathway $[15,16]$. In contrast, fMLP stimulation results in (PI3K/AKT)/mTOR pathway activation, leading to end-stream activation of NADPH oxidase followed by production and accumulation of ROS within the cytosol of the cell. Downstream steps post-activation of NADPH oxidase remain the same as in the PMA-activation pathway $[17,18]$. The results demonstrated that the amount of NETs generated by this co-stimulation is comparable to the amount of NETs generated by stimulation with fMLP alone. Furthermore, neutrophils stimulated with PMA alone produced significantly higher amounts of NETs when compared with fMLP- and PMA/fMLP-stimulated cells. Similar findings were observed when fMLP and CaI were combined to induce NETosis. While fMLP and fMLP/CaI stimulation resulted in the generation of similar amounts of NETs, the treatment of the cells with CaI-only resulted in significantly higher NET release by neutrophils. The first explanation of these phenomena can be attributed to ROS production, which plays a major role in NETosis intracellular activation pathways. Both PMA and fMLP activate cascades of intracellular events that eventually promote the generation of ROS within the cytosol, which in turn leads to NET formation. In PMNs, the MAP kinase-mediated transduction pathway can be activated by a range of stimuli and covers the activation of a variety of cellular functions, including regulation of proliferation, adhesion, cell migration, and respiratory bursts. MAP kinase, in its native state, exists in two distinct isoforms, ERK1 (42 kDa) and ERK2 (44 kDa). Both isoforms are regulated by the phosphorylation of specific tyrosine and threonine residues present within their structures. Data reported by Zhang et al. revealed that fMLP, PMA, and the $\mathrm{Ca}^{2+}$-ATPase inhibitors thapsigargin (Tg) and cyclopiazonic acid (CPA), can significantly increase MAP kinase activity. This study shows not only that ROS generation can be initiated in a PKCdependent (PMA) or PKC-independent (fMLP) manner, but also that an inhibition mechanism can block both pathways existing within the cell [19]. Accordingly, we can hypothesise that neutrophils possess a protecting mechanism that is triggered when the ROS concentration reaches non-physiological levels.

Stålhammar et al. reported that PMA alone promotes a noticeably increased oxidative burst when compared to a negative control. The magnitude of ROS generation after activation with PMA was comparable to that observed following neutrophil co-incubation with Escherichia coli strains. Interestingly, after pre-incubation of neutrophils with fMLP and then stimulation with either PMA or E. coli, the levels of generated ROS were significantly lower when compared to the no-fMLP pre-incubation step. In another set of assays, Stålhammar et al. implemented pre-incubation of neutrophils with IL-8 [14]. The results indicated that pre-incubation of human neutrophils with IL-8 prior to stimulation with either PMA or E. coli has no inhibitory effect on ROS generation in vitro, compared to the control group. There is no clear evidence as to why this inhibition occurs but it has been proposed that reduced respiratory bursts caused by fMLP might be a potential mechanism that reduces the detrimental effects of uncontrolled inflammation during neutrophil migration. Generally, low levels of NET release or lack of NETosis itself seems to be connected with low levels of intracellular ROS. Our data support this finding, with both fMLP/PMA and fMLP/CaI NET generation reported to be significantly lower compared to a single stimulation (PMA and CaI, respectively).

When the two most potent NETosis inducers (PMA and $\mathrm{CaI}$ ) were combined, the NET release was significantly lower than both single-PMA and single-CaI stimulations. It is worth noting that $\mathrm{CaI}$ is a mobile ion-carrier that actively transports calcium ions through the cell membrane. Initiation of NET release induced by $\mathrm{CaI}$ is different from PMA- or fMLP-mediated initiation [20]. CaI initiates extracellular $\mathrm{Ca}^{2+}$ influx, as well as mobilisation of $\mathrm{Ca}^{2+}$ from intracellular stores, which leads to the activation of tyrosine kinases and the generation of mtROS within the cell. In subsequent cascades mtROS activates nuclear transcription factor NFkB, which leads to NET generation [21]. NETosis is a complex process, and although NADPH oxidase activity and/or the presence of mtROS is required to initiate NETosis, additional steps are necessary to generate and release fully mature NETs. Gupta et al. have shown that an elevated intracellular level of calcium ions induces $\mathrm{Ca}^{2+}$-dependent PAD4 activity that leads to histone citrullination [22]. This process directly initiates the penultimate-step of NET formation, as was reported by Neeli et al. and Wang et al. [23, 24]. Contrary to these findings, our results reveal that elevated levels of calcium ion uptake by the cell after CaI stimulation efficiently diminish NETosis when additional activation with PMA occurs. This phenomenon could be related to the observation that, in the natural life cycle of the cell, calcium ions act downstream of PCK and elevated levels only occur midway through NETosis. As shown by Neeli et al., neutrophils initially primed with $\mathrm{CaI}$ and then stimulated with PMA exhibit diminished citrullination of histones [13]. Since histone citrullination allows chromatin to decondense and mix with granular content, double stimulation may result in inhibition of NETosis. This could explain the low NET yield observed after using this stimuli pairing. 
In our study, we observed a notable difference between NETosis induced by a single stimulus and that induced by a combination of them. In standard conditions, when PMA, fMLP, and CaI act alone, cells undergo NETosis or remain intact. In our experiments, cells stimulated with a combination of PMA/fMLP/CaI did not initiate classic NETosis. The amount of generated NETs was significantly lower when compared to other stimulations, whilst simultaneously the NET release was higher compared to negative controls. The microscopic visualisation of neutrophils stimulated with a combination of PMA, fMLP, and CaI revealed that there were few classical NET structures and that the morphology of the cell nuclei was different when compared to non-stimulated cells. The so-called "swollen nucleus" is one of the classical hallmarks of NETosis. This usually appears in very early stages after NETosis initiation and is mostly visible when nuclear chromatin starts to decondense and is ready to mix with cytoplasmic and granular proteins prior to being extruded into the extracellular matrix as mature NETs [25]. In our case this state lasted for $3 \mathrm{~h}$, indicating that activated neutrophils are suspended in a state somewhere between unresolved NETosis and classic programmed cell death. Martinod et al. attributed this state to arginine citrullination inhibition in histones, which prevents nucleosomes unravelling and consequently blocks chromatin from being expelled from the swollen nuclei [26].

Contrary to our findings, Neeli et al. showed that a combination of PMA and CaI is more effective at eliciting NETosis than is single stimulation. It is difficult to relate our data to this finding because concentrations of used stimuli were not published and the NET measurement was performed using a different technique. Interestingly, the authors were able to show that a combination of stimuli completely stopped histone citrullination (deimination), which is considered to be a crucial step in NET formation [13]. This clearly suggests that there are alternative pathways regulating NETosis, which are activated by co-stimulation.

In summary, our results indicate that co-stimulation of human PMNs with various NETosis inducers not only failed to enhance NET generation but also diminished it. This might be due to an internal safety mechanism protecting cells from generating ROS too rapidly, resulting in them entering apoptosis instead of NETosis. Our explanations of this phenomenon further emphasise that there are alternative signalling cascades involved when cells are co-stimulated. However, more in-depth studies are required in order to further explore this hypothesis.

\section{Acknowledgments}

This research was co-funded from resources received from the Polish National Science Centre upon covenant DEC-2011/03/N/NZ6/03652.

The authors declare no conflict of interest.

\section{References}

1. Kaplan MJ, Radic M (2012): Neutrophil extracellular traps: double-edged swords of innate immunity. J Immunol 189: 2689-2695.

2. Pruchniak MP, Kotula I, Manda-Handzlik A (2015): Neutrophil extracellular traps (Nets) impact upon autoimmune disorders. Centr Eur J Immunol 2: 217-224.

3. Yang H, Biermann MH, Brauner JM, et al. (2016): New Insights into Neutrophil Extracellular Traps: Mechanisms of Formation and Role in Inflammation. Front Immunol 7: 302.

4. Pruchniak MP, Arazna M, Demkow U (2013): Life of neutrophil: from stem cell to neutrophil extracellular trap. Respir Physiol Neurobiol 187: 68-73.

5. Branzk N, Papayannopoulos V (2013): Molecular mechanisms regulating NETosis in infection and disease. Semin Immunopathol 35: 513-530.

6. de Oliveira S, Rosowski EE, Huttenlocher A (2016): Neutrophil migration in infection and wound repair: going forward in reverse. Nat Rev Immunol 16: 378-391.

7. Zawrotniak M, Rapala-Kozik M (2013): Neutrophil extracellular traps (NETs) - formation and implications. Acta Biochim Pol 60: 277-284.

8. Garcia-Romo GS, Caielli S, Vega B, et al. (2011): Netting neutrophils are major inducers of type I IFN production in pediatric systemic lupus erythematosus. Sci Transl Med 3: 73 ra20.

9. Sur Chowdhury C, Giaglis S, Walker UA, et al. (2014): Enhanced neutrophil extracellular trap generation in rheumatoid arthritis: analysis of underlying signal transduction pathways and potential diagnostic utility. Arthritis Res Ther 16: R122.

10. Lee KH, Kronbichler A, Park DD, et al. (2017): Neutrophil extracellular traps (NETs) in autoimmune diseases: A comprehensive review. Autoimmun Rev 16: 1160-1173.

11. Shen XF, Cao K, Jiang JP, et al. (2017): Neutrophil dysregulation during sepsis: an overview and update. J Cell Mol Med 21: 1687-1697.

12. Gupta S, Kaplan MJ (2016): The role of neutrophils and NETosis in autoimmune and renal diseases. Nat Rev Nephrol 12: 402-413.

13. Neeli I, Radic M (2013): Opposition between PKC isoforms regulates histone deimination and neutrophil extracellular chromatin release. Front Immunol 4: 38.

14. Stålhammar ME, Douhan Hakansson L, Sindelar R (2017): Bacterial N-formyl Peptides Reduce PMA- and Escherichia coli-Induced Neutrophil Respiratory Burst in Term Neonates and Adults. Scand J Immunol 85: 365-371.

15. Cohen G, Makranz C, Spira M, et al. (2006): Non-PKC DAG/ phorbol-ester receptor(s) inhibit complement receptor-3 and nPKC inhibit scavenger receptor-AI/II-mediated myelin phagocytosis but cPKC, PI3k, and PLCgamma activate myelin phagocytosis by both. Glia 53: 538-550.

16. Papayannopoulos V, Metzler KD, Hakkim A, et al. (2010): Neutrophil elastase and myeloperoxidase regulate the formation of neutrophil extracellular traps. J Cell Biol 191: 677-691.

17. Itakura A, McCarty OJ (2013): Pivotal role for the mTOR pathway in the formation of neutrophil extracellular traps via regulation of autophagy. Am J Physiol Cell Physiol 305: C348-354.

18. Stoiber W, Obermayer A, Steinbacher P, et al. (2015): The Role of Reactive Oxygen Species (ROS) in the Formation of Extracellular Traps (ETs) in Humans. Biomolecules 5: 702-723. 
19. Zhang H, Garlichs CD, Mugge A, et al. (1998): Involvement of tyrosine kinases, $\mathrm{Ca} 2+$ and $\mathrm{PKC}$ in activation of mitogenactivated protein (MAP) kinase in human polymorphonuclear neutrophils. J Physiol 513 ( Pt 2): 359-367.

20. Hoppenbrouwers T, Autar ASA, Sultan AR, et al. (2017): In vitro induction of NETosis: Comprehensive live imaging comparison and systematic review. PLoS One 12: e0176472.

21. Dan Dunn J, Alvarez LA, Zhang X, et al. (2015): Reactive oxygen species and mitochondria: A nexus of cellular homeostasis. Redox Biol 6: 472-485.

22. Gupta AK, Joshi MB, Philippova M, et al. (2010): Activated endothelial cells induce neutrophil extracellular traps and are susceptible to NETosis-mediated cell death. FEBS Lett 584: 3193-3197.

23. Neeli I, Khan SN, Radic M (2008): Histone deimination as a response to inflammatory stimuli in neutrophils. J Immunol 180: 1895-1902.

24. Wang Y, Li M, Stadler S, et al. (2009): Histone hypercitrullination mediates chromatin decondensation and neutrophil extracellular trap formation. J Cell Biol 184: 205-213.

25. Neubert E, Meyer D, Rocca F, et al. (2018): Chromatin swelling drives neutrophil extracellular trap release. Nat Commun 9: 3767.

26. Martinod K, Demers M, Fuchs TA, et al. (2013): Neutrophil histone modification by peptidylarginine deiminase 4 is critical for deep vein thrombosis in mice. Proc Natl Acad Sci U S A 10: 8674-8679. 